\title{
Serological cross-reactivity of Trypanosoma cruzi, Ehrlichia canis, Toxoplasma gondii, Neospora caninum and Babesia canis to Leishmania infantum chagasi tests in dogs
}

\author{
Maurício Franco Zanette ${ }^{[1]}$, Valéria Marçal Felix de Lima ${ }^{[2]}$, Márcia Dalastra Laurenti ${ }^{[3]}$, \\ Claudio Nazaretian Rossi ${ }^{[1]}$, Juliana Peloi Vides ${ }^{[1]}$, Rafael Felipe da Costa Vieira ${ }^{[4]}$, \\ Alexander Welker Biondo ${ }^{[5],[6]}$ and Mary Marcondes ${ }^{[2]}$
}

\begin{abstract}
[1]. Pós-Graduação em Ciência Animal, Faculdade de Medicina Veterinária, Universidade Estadual Paulista, Araçatuba, SP. [2]. Departamento de Clínica, Cirurgia e Reprodução Animal, Faculdade de Medicina Veterinária, Universidade Estadual Paulista, Araçatuba, SP. [3]. Laboratório de Investigações Médicas, Faculdade de Medicina, Universidade de São Paulo, SP. [4]. Departamento de Ciências Veterinárias, Universidade Federal da Paraíba, Areia, PB. [5]. Departamento de Medicina Veterinária, Universidade Federal do Paraná, Curitiba, PR. [6]. Department of Veterinary Pathobiology, University of Illinois, Urbana, IL, USA.
\end{abstract}

\begin{abstract}
Introduction: The aim of this study was to evaluate the serological cross-reactivity between Leishmania sp. and other canine pathogens. Methods: Positive serum samples for Ehrlichia canis, Babesia canis, Toxoplasma gondii, Neospora caninum and Trypanosoma cruzi were tested using three serological methods enzyme linked immunosorbent assay (ELISA), indirect immunofluorescent antibody test (IFAT) and Kalazar Detect ${ }^{\mathrm{TM}}$, for canine visceral leishmaniasis. Results: Of the 57 dog samples tested, $24(42.1 \%)$ tested positive using one of the three serological methods: 10/57 (17.5\%) for ELISA, 11/57 (19.3\%) for IFAT and 3/57 (5.3\%) for Kalazar Detect ${ }^{\mathrm{TM}}$. Conclusions: Our results demonstrated that the presence of other infectious agents may lead to cross-reactivity on leishmaniasis serological tests.
\end{abstract}

Keywords: Diagnosis. Serology. Visceral leishmaniasis.

Visceral leishmaniasis (VL) is a widespread protozoan zoonosis caused by the genus Leishmania. Leishmania chagasi (Leishmania infantum) is the reported species present in the New World ${ }^{1}$. Dogs are considered the main epidemiological reservoirs of $\mathrm{VL}$ in the domestic environment, and mandatory euthanasia of positive dogs has been the basis of disease control in several developing countries, including Brazil ${ }^{2}$. Because the clinical diagnosis of canine visceral leishmaniasis (CVL) is not reliable due to the wide variety of clinical signs of CVL and the high percentage of asymptomatic dogs, serological methods have been used for the definitive diagnosis of CVL'1 .

Although the detection of amastigote forms of Leishmania sp. may be considered the gold standard for the diagnosis of $\mathrm{VL}$, this procedure is laborious and difficult to routinely apply under field conditions. Serological methods, such as the enzyme linked immunosorbent assay (ELISA) and the indirect immunofluorescent antibody test (IFAT), have been indicated as better, rapid and reliable diagnostic methods that are easily standardized and suitable for mass testing ${ }^{3}$. Despite the high sensitivity and specificity of serological assays for

\footnotetext{
Address to: Dra Mary Marcondes. Dept ${ }^{\mathrm{a}}$ Clínica, Cirurgia e Reprodução Animal/ FMV/UNESP. Rua Clóvis Pestana 793, 16050-680 Araçatuba, SP, Brasil.

Phone/Fax: 5518 3636-1415

e-mail: marcondes@fmva.unesp.br

Received 23 July 2012

Accepted 14 February 2013
}

canine leishmaniasis, the presence of cross-reactivity remains controversial. The aim of the present study was to evaluate the cross-reactivity of serum samples from dogs serologically reactive for Trypanosoma cruzi, Ehrlichia canis, Babesia canis, Toxoplasma gondii and Neospora caninum by ELISA, IFAT and an immunochromatographic test (Kalazar Detect ${ }^{\mathrm{TM}}$, InBios Inc., Seattle, WA, USA) for canine leishmaniasis. All dogs were from areas that were non-endemic for leishmaniasis, Chagas disease and Trypanosoma evansi, free of vectors and with no single reported case to date.

A total of fifty-seven serum samples were used: 14 dogs experimentally infected with $T$. cruzi (Colombian strain) with chronic cardiomyopathy and anti-T. cruzi IgG antibodies, as determined by IFAT, with titers ranging from 160 to $640^{4}$; 12 dogs naturally infected with $B$. canis and presenting pale mucous membranes, anemia, intraerythrocytic inclusions of Babesia sp. and anti-B. canis antibodies, as determined by IFAT, with titers ranging from 400 to $800^{5} ; 13$ dogs naturally infected with E. canis and presenting thrombocytopenia, Ehrlichia morulae within macrophages on capillary blood smears and antibodies, as detected by a commercially available kit (SNAP ${ }^{\circledR}$ 3Dx ${ }^{\circledR}$, IDEXX Laboratories Inc., Westbrook, ME, USA); 10 dogs naturally infected with $T$. gondii and presenting clinical signs of the disease and anti-T. gondii antibodies, as detected by IFAT, with titers ranging from 1:128 to $1: 1024^{6}$; and eight dogs presenting clinical signs of $N$. caninum infection, with anti$N$. caninum antibodies, no detected anti- $T$. gondii antibodies and IFAT antibody titers ranging from 400 to $800^{7}$. 
The serological detection of anti-Leishmania chagasi $\operatorname{IgG}$ antibodies was performed by ELISA as previously described ${ }^{8}$ using an L. chagasi total promastigote lysate (strain MHOM/ BR/74/PP75) and alkaline phosphatase-conjugated anti-dog IgG (Sigma-Aldrich, St. Louis, MO, USA). A cut-off value of 0.270 was determined using the analysis of serum samples from 50 healthy dogs from an area that was non-endemic for leishmaniasis (mean $+3 \mathrm{SD}$ ). The detection of $L$. chagasi antibodies by IFAT was determined as previously described ${ }^{9}$ using a suspension of parasites (MHOM/BR/72) in buffered saline solution (PBS) at a concentration of $8.1 \times 10^{6}$ promastigotes $/ \mathrm{mL}$ and a fluorescein isothiocyanate-conjugated anti-dog IgG (Sigma-Aldrich, St. Louis, MO, USA). The samples were considered positive when titers were $\geq 1: 40$. Positive sera were serially diluted and tested to establish the maximum reaction titer. The samples were also tested for Leishmania sp. using an immunochromatographic qualitative antibody assay against $L$. chagasi recombinant K39 (rK39) antigen (Kalazar Detect ${ }^{\mathrm{TM}}$, InBios Inc., Seattle, WA, USA) according to the manufacturer's protocol.

A total of $24 / 57(42.1 \%)$ dog samples tested positive using any of the three serological methods for Leishmania sp. (Table 1). Of the positive ELISA samples, the optical density (OD) values of the T. cruzi samples ranged from 0.299 to 0.941 , with an average of $0.531 \pm 0.226$, and the OD value of the $E$. canis sample was 0.385 (Figure 1). Of the positive IFAT samples, the titer values ranged from 40 to 160 and from 40 to 80 for the T. cruzi and T. gondii samples, respectively. Of the positive dipstick samples, the number of samples that crossreacted using this method was lower than those observed using the ELISA and IFAT methods (Table 1).

Although the ELISA and IFAT tests were conducted using Leishmania crude antigens and although the observed cross-reactivity may be explained by a decrease in test specificity $^{2}$, a previous study also used crude antigens and observed no cross-reactivity with E. canis, T. gondii and B. canis $^{8}$. Antibodies against $T$. cruzi have been recognized using conventional serological methods as the main cause of crossreactivity with Leishmania due to the phylogenetic similarity between Leishmania sp. and Trypanosoma cruzi, which poses

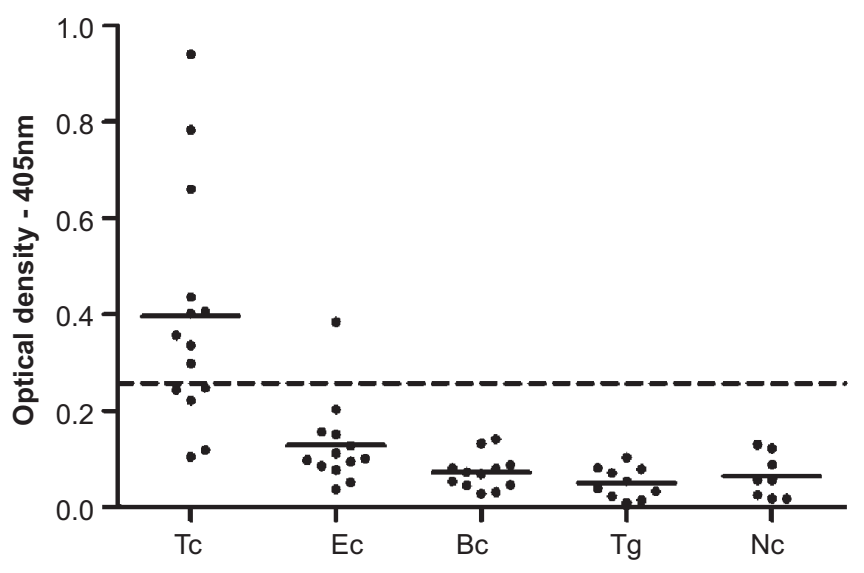

FIGURE 1 - Distribution of individual enzyme linked immunosorbent assay (ELISA) results for the diagnosis of Leishmania chagasi in 57 seropositive dogs with other infectious and parasitic agents: 14 with Trypanosoma cruzi (Tc), 13 with Ehrlichia canis (Ec), 12 with Babesia canis (Bc), ten with Toxoplasma gondii (Tg), and eight with Neospora caninum $(\mathrm{Nc})$. The horizontal line within each data group is the arithmetic mean. The horizontal line at 0.270 represents the cut-off optical density value.

a problem for overlapping endemic areas ${ }^{10,11}$. In the present study, T. cruzi displayed a high percentage of cross-reactivity with Leishmania when using ELISA and IFAT, both of which are tests that are used as standard diagnostic methods for CVL in Brazil. Samples from five dogs cross-reacted with T. cruzi in both the ELISA and the IFAT. The dipstick test was the only test displaying no cross-reactivity between T. cruzi and Leishmania sp., confirming that rK39 does not cross-react with T. cruzi ${ }^{12}$.

The identification of ELISA cross-reactivity with $E$. canis $(1 / 13 ; 7.7 \%)$ corroborated previous studies that reported $2 / 3$ $(66.6 \%)$ cross-reactivity on the IFAT and $1 / 3(33.3 \%)$ crossreactivity on the ELISA ${ }^{13}$. Although positive, titers were within 2 -fold of the cut-off value (OD value 0.385 ), and these samples tested negative by IFAT. None of the 12 Babesia canis samples

TABLE 1 - Results obtained by ELISA, IFAT and Kalazar Detect ${ }^{\mathrm{TM}}$ for the detection of cross-reactivity between Leishmania chagasi and other infectious and parasitic agents in dog serum samples.

\begin{tabular}{|c|c|c|c|c|c|c|c|}
\hline \multirow{3}{*}{ Infectious/parasitic agents } & \multicolumn{7}{|c|}{ Positive dogs (n/\%) } \\
\hline & \multirow[b]{2}{*}{$\mathrm{n}$} & \multicolumn{2}{|c|}{ ELISA } & \multicolumn{2}{|c|}{ IFAT } & \multicolumn{2}{|c|}{ Kalazar Detect $\mathrm{T}^{\mathrm{TM}}$} \\
\hline & & $\mathrm{n}$ & $\%$ & $\mathrm{n}$ & $\%$ & $\mathrm{n}$ & $\%$ \\
\hline Trypanosoma cruzi & 14 & 9 & 64.3 & 6 & 42.9 & 0 & 0.0 \\
\hline Ehrlichia canis & 13 & 1 & 7.7 & 0 & 0.0 & 1 & 7.7 \\
\hline Babesia canis & 12 & 0 & 0.0 & 0 & 0.0 & 0 & 0.0 \\
\hline Neospora caninum & 8 & 0 & 0.0 & 0 & 0.0 & 1 & 12.5 \\
\hline Total & 57 & \multicolumn{2}{|c|}{10} & \multicolumn{2}{|c|}{11} & \multicolumn{2}{|c|}{3} \\
\hline
\end{tabular}

ELISA: enzyme linked immunosorbent assay; IFAT: indirect immunofluorescent antibody test. 
displayed cross-reactivity with Leishmania by either ELISA or IFAT, as previously demonstrated ${ }^{8}$.

In a previous study, cross-reactivity between Leishmania sp. and $T$. gondii was not identified when dog serum samples were tested by ELISA and IFAT ${ }^{13}$. Although IFAT is considered highly specific $^{3}$, the present study has demonstrated that half of the dogs $(5 / 10 ; 50 \%)$ with anti-T. gondii antibodies were mistakenly considered serologically positive for visceral leishmaniasis.

Despite the fact that the dipstick test did not display crossreactivity with Chagas disease, dogs seroreactive for $E$. canis $(1 / 13 ; 7.7 \%)$, T. gondii $(1 / 10 ; 10 \%)$, and $N$. caninum $(1 / 8 ; 12.5 \%)$ induced false positive results on this test. Our results differed from those of a previous study that reported 3/12 (25\%) samples cross-reacting with Chagas disease, and our results differed from those of a previous study, which found 3/12 (25\%) crossreacted with Chagas disease and no false positives with $T$. gondii, although 1/3 (33.3\%) dogs with ehrlichiosis presented false positive results on the dipstick ${ }^{14}$. Although the dipstick test has been developed for the diagnosis of visceral leishmaniasis in humans, previous studies have demonstrated that the sensitivity and specificity of this test ranged from $83 \%$ to $91.5 \%$ and from $94.7 \%$ to $100 \%$, respectively, for disease diagnosis in $\operatorname{dogs}^{8,14}$. False positive results were also observed in a rapid commercial ELISA test for dog leishmaniasis, with cross-reactivity found in 3/6 samples (50\%) of T. gondii, 1/2 (50\%) samples of $T$. gondii and $N$. caninum, 3/4 (75\%) samples of $B$. canis and $E$. canis and 4/7 samples (57.1\%) of T. cruzi ${ }^{15}$. Thus, rapid inclinic diagnostic tests should be used in association with other diagnostic tests and should be used carefully when screening for canine visceral leishmaniasis in areas that are endemic for ehrlichiosis, babesiosis, toxoplasmosis and neosporosis. In the present study, with the sole use of the ELISA, IFAT or commercial immunochromatographic tests, approximately $19 \%$, $14 \%$ and $11 \%$ of the dogs tested would have been considered positive for leishmaniasis, respectively. A recent guideline consensus has concluded that dogs should be considered infected by serology only when antibody titers are 2- to 4-fold higher than the positive threshold value. Otherwise, cytology and polymerase chain reaction (PCR) using bone marrow and/or lymph node samples should be conducted to accurately confirm the diagnosis ${ }^{11}$.

Despite the territorial expansion of VL in Brazil, where the disease has reached even the southern states of the country, when the present study was conducted, the number of municipalities in which vectors and reported cases were present was lower than the current number of such municipalities, and many areas were still considered to be disease-free. Thus, the possibility of dogs having been infected prior to the time of this study by Leishmania chagasi (L. infantum) is remote.

In summary, our results indicate that confounding infectious agents such as Trypanosoma cruzi, Ehrlichia canis, Babesia canis, Toxoplasma gondii and Neospora caninum may cross-react with leishmaniasis serological tests and lead clinicians to falsely diagnose visceral leishmaniasis. Thus, the use of serological methods with low antibody titers for the diagnosis of Leishmaniainfected dogs as a criterion for euthanasia should be reassessed.

\section{ACKNOWLEDGMENTS}

The authors thank Dr. Solange Maria Gennari, Dr. Mitika Kuribayashi Hagiwara and Dr. Antonio Aparecido Camacho for providing dog serum samples.

\section{CONFLICT OF INTEREST}

The authors declare that there is no conflict of interest.

\section{FINANCIAL SUPPORT}

Fundação de Amparo a Pesquisa do Estado de São Paulo, FAPESP (Process 04/10872-6).

\section{REFERENCES}

1. Baneth G. Leishmaniasis. In: Greene CE, editor. Infectious diseases of the dog and cat. $3^{\text {rd }}$ ed. Philadelphia: Elsevier; 2006. p. 685-698.

2. Ikeda-Garcia FA, Feitosa MM. Métodos de diagnóstico da leishmaniose visceral canina. Rev Clin Vet 2006; 62:32-38.

3. Távora MPF, Pereira MAVC, Silva VL, Vita GF. Estudo de validação comparativo entre as técnicas Elisa e RIFI para diagnosticar Leishmania sp. em cães errantes apreendidos no município de Campos dos Goytacazes, Estado do Rio de Janeiro. Rev Soc Bras Med Trop 2007; 40:482-483.

4. Alvarez M, Cerisola JA, Rohwedder RW. Test de inmunoflorescencia para el diagnóstico de la Enfermedad de Chagas. Bol Chileno Parasitol 1968; 23:4-9.

5. Trapp SM, Dagnone AS, Vidotto O, Freire RL, Amude AM, Morais HA Seroepidemiology of canine babesiosis and ehrlichiosis in a hospital population. Vet Parasitol 2006; 140:223-230.

6. Camargo ME. Introdução às técnicas de imunofluorescência. Rev Bras Patol Clin 1974; 10:143-171.

7. Dubey JP, Carpenter JL, Speer CA, Topper MJ, Uggla A. Newly recognized fatal protozoan disease of dogs. J Am Vet Med Assoc 1988; 192:1269-1285.

8. Lima VMF, Biazzono L, Silva AC, Correa APFL, Luvizotto MCR. Serological diagnosis of visceral leishmaniasis by an enzyme immunoassay using protein A in naturally infected dogs. Pesq Vet Bras 2005; 25:215-218.

9. Nardo CDD, Rossi CN, Laurenti MD, Marcondes M. Anti-Leishmania infantum syn chagasi antibodies in dogs from São José do Rio Preto, São Paulo. Braz J Vet Res Anim Sci 2011; 48:425-428.

10. Umezawa ES, Souza AI, Pinedo-Cancino V, Marcondes M, Marcili A, Camargo LMA, et al. TESA-blot for the diagnosis of Chagas disease in dogs from co-endemic regions for Trypanosoma cruzi, Trypanosoma evansi and Leishmania chagasi. Acta Trop 2009; 111:15-20.

11. Paltrinieri S, Solano-Gallego L, Fondati A, Lubas G, Grandoni L, Castagnaro M, et al. Guidelines for diagnosis and clinical classification of leishmaniasis in dogs. J Am Vet Med Assoc 2010; 236:1184-1191.

12. Carvalho SFG, Lemos EM, Corey R, Dietze R. Performance of recombinant k39 antigen in the diagnosis of Brazilian visceral leishmaniasis. Am J Trop Med Hyg 2003; 68:321-324.

13. Ferreira EC, Lana M, Carneiro M, Reis AB, Paes DV, Silva ES, et al. Comparison of serological assays for the diagnosis of canine visceral leishmaniasis in animals presenting different clinical manifestations. Vet Parasitol 2007; 146:235-241.

14. Lemos EM, Laurenti MD, Moreira MAB, Reis AB, Giunchetti RC, Raychaundhuri $\mathrm{S}$, et al. Canine visceral leishmaniasis: performance of a rapid diagnostic test $\left(\right.$ Kalazar $^{\mathrm{TM}}$ Detect $^{\mathrm{TM}}$ ) in dogs with and without signs of the disease. Acta Trop 2008; 107:205-207.

15. Marcondes M, Biondo AW, Gomes AAD, Silva ARS, Vieira RFC, Camacho AA, et al. Validation of a Leishmania infantum ELISA rapid test for serological diagnosis of Leishamania chagasi in dogs. Vet Parasitol 2011; 175:15-19. 